\title{
Refinement and Validation of the Balanced Inventory of Mindfulness-related Skills
}

(BIMS)

Stephanie S Padmanabham1, Zarina R. Bilgrami2, Tory Eisenlohr-Moul3, Nicholas T. Van Dam1,2*

1 Melbourne School of Psychological Sciences, University of Melbourne, Melbourne, Australia

2 Department of Psychiatry, Icahn School of Medicine at Mount Sinai, New York, NY, USA 3 Department of Psychiatry, University of Illinois at Chicago, Chicago, IL, USA

Disclosure of Potential Conflict of Interest: The authors declare that they have no conflicts of interest.

Research involving human participants and/or animals: All reported studies/

experiments with human subjects performed by the authors complied with all applicable ethical standards (including the Helsinki declaration and its amendments, institutional/national research committee standards, and international/national/institutional guidelines)

Informed Consent: All human subjects provided informed consent prior to participation.

*To Whom Correspondence Should be Addressed:

Dr. Nicholas Van Dam

Melbourne School of Psychological Sciences

University of Melbourne

Melbourne, VIC, 3010 Australia 


\begin{abstract}
Objectives: The goal of the current study was to refine and validate a revision of the Five Facet Mindfulness Questionnaire (FFMQ), with the goal of retaining its useful features and mitigating those features that have been identified to be problematic.

Methods: A 30-item pilot version of the Balanced Inventory of Mindfulness-related Skills (BIMS) was developed by implementing the structured alternative item format (presenting both positive and negative aspects of each item) and revising the wording of items. Herein, we collected data from a convenience sample of $n=1,014$ individuals, reduced to $n=757$ after data cleaning. We conducted exploratory factor analysis (EFA) and confirmatory factor analyses (CFA) on randomly separated subsets of the sample and explored response patterns and correlations with relevant measures (including a short-form of the FFMQ).
\end{abstract}

Results: Parallel analysis and EFA indicated a five-factor, correlated structure across a final 27 items (omitting 3 items due to poor fit), confirmed by CFA. A full sample CFA using an asymptotically distribution free fit function indicated excellent model fit (CFI/TLI > 0.99, RMSEA $<0.05$, SRMR $<0.05)$. The BIMS scales exhibited strong correlations with the FFMQ subscales and related scales. The scale did not, however, reduce correlations with social desirability, which has been shown to be associated with method effects.

Conclusions: The BIMS represents a psychometrically sound revision of the FFMQ that retains the five-factor structure of the original scale while eliminating method effects. It is strongly correlated with the original scale and exhibits comparable correlations with attentional control, emotion regulation, and personality characteristics. Critically, the BIMS represents a measure of mindfulness-related skills, shifting the focus to clinically relevant constructs that may interact but do not necessarily sum to a unitary trait.

Keywords: Mindfulness, Psychometrics, Assessment, FFMQ, BIMS 
Data Availability Statement: Data are available at the Open Science Framework (https://osf.io/jdk7h/)

Mindfulness, the awareness that results from purposeful attention while being in the present moment with the maintenance of a non-judgmental perspective (Kabat-Zinn, 2013), has become in an incredibly popular construct over the past three decades Van Dam, van Vugt, et al., 2018). Research on mindfulness relies heavily on use of self-report measures (see e.g., Baer 2019), reflecting a trend in psychology more generally (Baumeister et al., 2007). Given such emphasis on self-report, especially in relation to the construct of mindfulness, it is critical that self-report measures are reliable and valid.

The Five Facet Mindfulness Questionnaire (FFMQ; Baer et al., 2006) is arguably one of the most popular measures of "trait" mindfulness. Per citation count (Van Dam, van Vugt, et al., 2018), it is the second most commonly used measure, behind the Mindful Attention Awareness Scale (MAAS; Brown \& Ryan, 2003). Despite its popularity, numerous authors have expressed concern about the psychometric properties and construct validity of the scale. Against the recommendations of the original study ( Baer et al., 2006), a total score is commonly presented. While many studies have replicated the original five-factor hierarchical structure (e.g., Christopher et al., 2012; de Bruin et al., 2012; Michalak et al., 2016; Veehof et al., 2011), others have failed to confirm a five-factor hierarchical structure or have identified better fitting alternative models (e.g., Lecuona et al., 2019; Lilja et al., 2011; Morgan et al., 2017; Tran et al., 2013; Van Dam et al., 2012; Williams et al., 2014). Yet others have found critical differences on the FFMQ as a whole or on its subscales between different samples (e.g., Baer et al., 2006; Karl et al., 2020; Van Dam et al., 2009; Williams et al., 2014). Numerous other studies have proposed alterations or short forms necessary for improved model-to-data fit (Bohlmeijer et al., 2011; Tran et al., 2013; Truong et al., 2020; Gu et al., 2016; Medvedev et al., 2017).

Despite concerns about the scale's properties, it does have clinical utility, mediating outcomes in mindfulness-based interventions (e.g., Völlestad et al., 2011), predicting long-term 
symptoms (e.g., Petrocchi \& Ottaviani, 2016), moderating relationships between vulnerability factors and mental health symptoms (e.g., Barnhofer et al., 2011), and showing important relationships to behaviors such as substance use (e.g., Eisenlohr-Moul et al., 2012). Operationalization of mindfulness is critical to evidence-based clinical practice (Baer, 2011); therefore, it is important to address measurement concerns about the FFMQ, leaving questions and disagreements about its content validity aside for the time-being (Grossman \& Van Dam, 2011; Baer, 2019).

Differences in scale properties among different backgrounds or cultures may be difficult to resolve (see e.g., Christopher et al., 2009; Karl et al., 2020). Similarly, whether mechanistic changes related to mindfulness-based interventions can actually be tracked by self-report mindfulness scales requires more detailed consideration (see e.g., Goldberg et al., 2016; Hsiao et al., 2019). Nonetheless, there are pragmatic issues that can be addressed to improve mindfulness measurement. Past work has suggested the FFMQ may exhibit method effects (Karl et al., 2020; Lecuona et al., 2019; Van Dam et al., 2012), which may or may not be related to desirability, another proposed issue with mindfulness measures (Grossman, 2008).

Method effects occur when the measurement of a given construct is impacted by the method used to administer a measure (Campbell \& Fiske, 1959). For example, one may administer self-report and behavioral measures of self-regulation separately, finding that the type of task (paper-and-pencil self-report vs. computer task) differentially influences the trait estimate for a given individual (see e.g., Enkavi et al., 2019). The presence of method effects may have a confounding influence on results, obscuring actual latent values and altering relationships to other variables (Campbell \& Fiske, 1959; Podskaoff et al., 2003). Method effects due to the wording directionality of questionnaire items are common among self-report measures. In such cases, items worded similarly (e.g., positive: I feel good, I am feeling pleasant; negative: I am unhappy, I am feeling down) may exhibit high correlations, in part, because of their wording and 
not their content (see DiStefano \& Motl, 2006). Method effects models raise concern because the construct of interest (e.g., self-esteem, depression, mindfulness) is not the only thing influencing how a participant answers an item; some participants may be more inclined than others to answer negative items negatively and positive items positively (see e.g., Van Dam et al., 2009). In some cases, there have been direct relationships between social desirability and method effects (e.g., Rauch et al., 2007). Such possibilities are particularly troubling for measures of mindfulness due to concern that people are responding to self-report items consistent with a desire to be mindful rather than a reliable appraisal of actual levels of mindfulness (see e.g., Grossman, 2008).

Mitigation of the above concerns may be achieved by changing the presentation and response format for items from the FFMQ. Previous efforts to mitigate method effects have tried to balance the number of positively and negatively worded items (e.g., Hart et al., 2015; Höfling et al., 2011) but an alternative approach (Harter \& Messer, 2012) is to provide two anchor statements of opposing directions (e.g., "Some adults find it hard to establish intimate relationships" vs. "Other adults do not have difficulty establishing intimate relationships"). Among other things, this "structured alternative format" is believed to normalize the response options in that it implies that people are generally like both of the anchors and that one anchor is not necessarily better than the other (see Harter \& Messer, 2012). The structured alternative format presents each item as a choice between two opposing statements with the goal of balancing desirability effects by normalizing both ends of a spectrum. Previous work with the structured alternative format has shown that it can dramatically reduce social desirability and has potential to mitigate method effects (Harter \& Messer, 2012).

While efforts are being made to improve scale properties, it is also worth considering the impact of middle-response options on scales measuring constructs that participants find confusing. The FFMQ uses a 5-point Likert-style response scale, which gives individuals the opportunity to endorse 3 - sometimes true. The middle response option is meant to represent 
neutrality or an indication of ambivalence (i.e. 'neither disagree nor agree'; Kulas et al., 2008). Despite its intention, there are instances where it is used as a 'dumping ground' when participants are unsure how to respond (Kulas et al., 2008). Moreover, middle category endorsement has been found to be negatively related with item clarity (Kulas \& Stachowski, 2009). Hence, unfamiliarity and confusion may lead to endorsement of middle response options in mindfulness questionnaires. Since some items on the FFMQ (and indeed, the concept of mindfulness itself) can be counterintuitive to the average Western reader (see e.g., Grossman \& Van Dam, 2011), this weakness of the response scale may be important issue to address.

The goal of the present work was to modify the FFMQ to account for many of the issues raised above. Our goal was not to generate a scale that reliably measures the concept of Mindfulness in the traditional Buddhist sense (Grossman \& Van Dam, 2011) but rather to improve a scale that is widely used to capture mindfulness-related skills commonly taught in contemporary psychotherapies (e.g., FFMQ), mitigating potential psychometric limitations and response biases. As such, we created a revision of the FFMQ, called the Balanced Inventory of Mindfulness-related Skills (BIMS) by introducing the structured alternative response format (see (Harter \& Messer, 2012) with both positive and negative statements for each item, as well as using an even numbered response scale for 30 revised items from the FFMQ (see Van Dam, Bilgrami, \& Eisenlohr-Moul, 2018). The goals of the present work were to (1) investigate the psychometric properties of the BIMS - aiming for a 5-factor model consistent with the FFMQ, (2) examine whether the BIMS exhibited convergent/divergent validity relative to the FFMQ, (3) examine the potential changes in associations of social desirability of the BIMS relative to the FFMQ, and (4) examine whether scale alteration could minimize previously observed method effects on the FFMQ. In order to obtain these goals, we collected several self-report measures, including the BIMS. To examine convergent and divergent validity, we examined emotion regulation and attentional control (both proposed to be fundamentally related to mindfulness and 
meditation; see Hölzel et al. 2011), as well as personality (certain aspects of which has shown strong association in meta-analysis; Giluk, 2009). We also included a measure of social desirability to explore associations as suggested (Grossman, 2008).

\section{Methods}

\section{Participants}

Participants were recruited via Amazon's Mechanical Turk (MTurk) with the requirement that they were American and were aged 18 to 60 years old; these inclusion criteria were implemented to reduce sample heterogeneity and minimize possible cultural effects on scale properties. Participants were reimbursed at a rate of US $\$ 7.50 /$ hour.

\section{Procedure}

After accepting the human intelligence task (HIT) on MTurk, participants were redirected to a Qualtrics webpage. Participants were required to complete a battery of questionnaires consisting of background information, various measures , three consistency check questions, and two bot response questions. They were then randomized to one to two conditions: (1) $75 \%$ of participants were given the BIMS prior to the FFMQ-SF or (2) $25 \%$ of participants were given the FFMQ-SF before the BIMS. The rationale for this 3:1 ratio was that we did not want any potential carryover effects from the FFMQ-SF to influence our ability to examine the properties of the BIMS. The randomly selected measure was presented after demographics and prior to all other self-report measures, while the other non-selected measure was presented after all other self-report measures.

\section{Measures}

Attentional Control Scale (ACS; Derryberry \& Reed, 2002). The ACS measures individual differences in attentional control. It consists of 20 items that are scored on a four-point scale: 1 (almost never) to 4 (always). A total score is obtained by summing all the items, where higher scores indicates higher attentional control. Example items include, "I can quickly switch 
from one task to another" and, "It's very hard for me to concentrate on a difficult task when there are noises around". In the present sample $(n=787)$, internal consistency was $\operatorname{good}(\alpha=0.89, \Omega=$ $0.89)$.

Balanced Inventory of Desirable Responding (BIDR-SF; Hart et al., 2015). The BIDRSF is a 16-item questionnaire that measures socially desirable responding. It consists of two subscales, self-deceptive enhancement (SDE) and impression management (IM), each of which had 8 items. Each item is rated on a seven-point scale ranging from 1 (not true) to 7 (very true). An example item from the SDE scale is, "I always know why I like things". An example item from the IM subscale is, "When I hear people talking privately, I avoid listening". In the present sample ( $\mathrm{n}=787$ ), internal consistency was reasonable (SDE: $\alpha=0.80, \Omega=0.80$; IM: $\alpha=0.79, \Omega$ $=0.80$ ). The scale has previously exhibited high test-retest correlations (IM: $r=.74$; SDE: $r$ $=.79$; Hart et al. 2015).

Balanced Inventory of Mindfulness-related Skills (BIMS). The BIMS was designed to address previously identified issues with the FFMQ (see Van Dam, Bilgrami, \& Eisenlohr-Moul, 2018; https://osf.io/wjz36/). The BIMS was designed to balance previously observed tendencies among non-meditators to reject mindfulness-absent items more than to endorse mindfulnesspresent items (see Van Dam et al., 2009). We initially used on the 26 FFMQ items identified by Soler and colleagues (2014) to maximally differentiate meditators from non-meditators. We revised these items via team consensus to create positive (e.g., "I can easily describe my feelings) and negative poles (e.g., "Sometimes I struggle to describe my feelings") and for clarity of wording. The item format used was the "structured alternative format" (Harter \& Messer, 2012), which has been shown to minimize method effects and normalize response patterns. While the structured alternative format can potentially increase item confusion (see Kalmet \& Fouladi, 2008), it has much potential to normalize response patterns and mitigate method effects (see Harter \& Messer, 2012). The preliminary version did not achieve satisfactory response 
properties (see Van Dam, Bilgrami, \& Eisenlohr-Moul, 2018), so we increased the number of items, selecting additional items to ensure 6 items from each of the 5 subscales of the original FFMQ. While this work was previously drafted in a preprint (https://mindrxiv.org/8qdzg/) for peer review, we lost confidence in a major aspect of our analytic approach (namely, what we now consider to be inappropriate use of the BiFactor model and subsequent reliance on an Item Response Theory implementation of that model). Participants were instructed to consider which of the two options was most characteristic of them and then rate the extent to which it was so. For the left option, the ratings ranged from 1 (very characteristic of me) to 3 (a little characteristic of me). For the right option, the ratings ranged from 4 (A little characteristic of me) to 6 (very characteristic of me). For all items, the positively worded mindfulness option was located on the right. Thus, higher scores indicated higher mindfulness-related skills.

Big Five Inventory $2^{\text {nd }}$ Edition - Short Form (BFI-2 - SF; Soto \& John, 2017). The BFI-2 - SF is a 30-item measure that measures individual differences in personality via the big-5 personality trait domains and facet scales. Items are scored on a five-point scale ranging from 1 (disagree strongly) to 5 (agree strongly). In the present sample $(\mathrm{n}=787)$, internal consistency was reasonable for all five scales (Open-mindedness: $\alpha=0.80, \Omega=0.81$; Conscientiousness: $\alpha=$ $0.83, \Omega=0.83$; Extraversion: $\alpha=0.77, \Omega=0.78$; Agreeableness: $\alpha=0.79, \Omega=0.80$; Negative Emotionality: $\alpha=0.87, \Omega=0.87$ ).

Conners Adult ADHD Rating Scale - Inconsistency Index (CAARS; Conners et al., 1999). The CAARS-Inconsistency Index was used to identify inconsistent responding within the battery of questionnaires. It consists of eight pairs of items that have shown very high correlations $(|r|>0.6)$ among item pairs in past research. The items were scored on a four-point scale ranging from 0 (not at all, never) to 3 (very much, very frequently). The CAARS consists of 8 item pairs, among each of which absolute difference scores are calculated after reverse scoring. Difference scores are summed across all item pairs, and a cut-off is applied. As 
recommended by the original work, inconsistency scores $>8$ were considered problematic. To ensure that inconsistency was measured within question blocks and across the questionnaire, the items were split into two question blocks. Some item pairs were presented in the same question block (to measure inconsistency within the same question block), and some item pairs were split across two question blocks (to measure inconsistency across the questionnaire

Difficulties in Emotion Regulation Scale - Short Form (DERS-SF; Kaufman et al., 2016). The DERS-SF is an 18-item questionnaire that assesses emotion regulation deficits. The DERS-SF consists of six subscales: awareness, clarity, goals, impulse, non-acceptance, and strategies. Items are scored on a five-point scale: 1 (almost never) to 5 (almost always). Subscale scores are obtained by summing the items scores for each subscale. The total score is obtained by summing the items. Greater difficulty with emotion regulation is reflected by higher scores. An example item from each subscale follow, Strategies: "When I'm upset, it takes me a long time to feel better", NonAcceptance: "When I'm upset, I feel guilty for feeling that way", Impulse: "When I'm upset, I become out of control”, Goals: "When I'm upset, I have difficulty concentrating", Awareness: "I pay attention to how I feel.", Clarity: "I have no idea how I am feeling”. In the present sample ( $\mathrm{n}=787)$, internal consistency was good for most subscales (Strategies: $\alpha=0.87, \Omega=0.87$; NonAcceptance: $\alpha=0.87, \Omega=0.87$; Impulse: $\alpha=0.91, \Omega=$ 0.91; Goals: $\alpha=0.91, \Omega=0.91$; Awareness: $\alpha=0.75, \Omega=0.75$; Clarity: $\alpha=0.87, \Omega=0.87$ ).

Five Factor Mindfulness Questionnaire - Short Form (FFMQ-SF; Bohlmeijer et al., 2011). The FFMQ-SF is a 24 -item questionnaire that measures mindfulness via five facets: observing, describing, acting with awareness, non-judging, and nonreactivity. All facets consisted of five items except for observe (four items). Items are rated on a five-point Likert scale; 1 (never or very rarely true) to 5 (very often or always true). Facet scores are obtained by adding up scores on individual items, where higher scores indicate greater mindfulness. An example item from each subscale follow, Describe: "I'm good at finding the words to describe 
my feelings", ActAware: "I find it difficult to stay focused on what's happening in the present moment”, NonJudge: “I tell myself that I shouldn't be feeling the way that I'm feeling”, NonReact: "I watch my feelings without getting carried away by them", Observe: "I notice the smells and aromas of things". In the present sample $(\mathrm{n}=787)$, internal consistency was good for most subscales (Describe: $\alpha=0.87, \Omega=0.87$; ActAware: $\alpha=0.89, \Omega=0.89$; NonJudge: $\alpha=$ $0.88, \Omega=0.89$; NonReact: $\alpha=0.86, \Omega=0.86$; Observe: $\alpha=0.79, \Omega=0.79$ ).

\section{Data Analysis Plan}

Data cleaning was done in RStudio (v. 1.1.463) using R (v. 3.5.3) and revolved largely around individual subject response patterns for and properties of the BIMS. Incomplete data was first removed, followed by elimination of any participants who reported non-compliance with instructions. Checks were conducted for careless responding via long-string responses and intraindividual response variability (IRV; Dunn et al., 2008). These analyses were implemented via the 'careless' package (v. 1.1.3). Eight or more consecutive responses of the same option were considered a long-string; participants who exhibited this pattern were excluded from further analysis ( Johnson, 2005). For IRV checks, 10,000 simulations of random responses (range: one to six) were generated to determine an upper bound of variability (99\%). Missing value analysis were carried out as a check to ensure that no cases of missing data was overlooked. Univariate checks were conducted where z-scores $>|3.289|$ for BIMS items were considered as univariate outliers. Checks for multivariate outliers were conducted flagging cases with Mahalanobis distance values of $p<.001$. Furthermore, participants that failed any of the checks implemented within the questionnaire were excluded from analysis. These checks included completion checks, bot response questions, CAARS-Inconsistency index measure, and attention checks. Participants were also excluded if they indicated that their data should be omitted due to their inability to focus on the task at hand (for example, being intoxicated while completing the measures). In summary, participants were excluded for incomplete data, self-report of non- 
compliance, longstring $>8$, IRV $>99 \%$, Univariate outliers, Multivariate outliers, or failure of various check measures.

The following packages were used in R via RStudio for psychometric analyses: lavaan (v. 0.6-3), nFactors (v. 2.3.3), psych (v. 1.8.12), and SemPlot (v. 1.1.1). The full dataset was randomly divided into exploratory $(n=450)$ and confirmatory samples $(n \cong 300)$. Exploratory factor analysis (EFA) and confirmatory factor analysis (CFA) were run on these samples, respectively. Prior to conducting EFA, we used modified Parallel Analysis (PA; Horn, 1965; Drasgwo \& Lissak, 1983), which implements principal axis factoring on unrotated components/factors to ascertain optimal number of factors. PA was implemented with 5000 permutations of existing data, comparing the extracted eigenvalues by component from the raw data to the $95^{\text {th }}$ percentile from the permuted data. During EFA, maximum likelihood estimation was implemented with promax rotation, using the factor number extracted from PA. Factor loadings less than 0.25 were ascertained to provide insufficient evidence of factor representation. Items exhibiting significant cross-loadings (i.e., no single factor loading $\geq 2 \mathrm{x}$ loading on another factor) were deleted (Costello \& Osborne, 2005). CFA models were fit using the non-robust variant of weighted least squares- mean and variance adjusted (WLSMV) estimator in lavaan (implemented as Diagonally Weighted Least Squares) (Rosseel, 2012). A Weighted Least Squares estimator was used due to the ordinal nature of the questionnaire items; the assumption of continuous and multivariate normality of observed variables required for maximum likelihood estimation was determined to be potentially inappropriate for the given measure. WLS is considered optimal for ordinal data and does not have such requirements of continuity and/or multivariate normality in distribution $(\mathrm{Li}, 2016)$. Criteria for a good model fit followed suggestions of a comparative fit index $(\mathrm{CFI})>0.95$, Tucker-Lewis Index $(\mathrm{TLI})>0.95$, standardized root mean square residual $(\mathrm{SRMR})<0.08$, and root mean square of approximation (RMSEA) $<0.06$ (Hu \& Bentler, 1999). 
Correlations were computed on the full dataset and separately for those individuals who received the FFMQ-SF and BIMS in opposite sequences. Between-scale and subscale correlations were also computed. To examine the potential implications of the revisions undertaken to create the BIMS, the BIMS and FFMQ-SF subscales were correlated with mindfulness-related measures. To investigate associations with desirable responding and to explore whether the revisions undertaken in the BIMS could mitigate desirable responding, the BIMS and FFMQ-SF were correlated with the BIDR-SF scales.

As not all items on the BIMS are represented on the FFMQ-SF, we computed scores for FFMQ analogue items from openly available data (Pang \& Ruch, 2019) and made comparisons between the BIMS, FFMQ-SF, and FFMQ. Item means for the BIMS and the corresponding items on the FFMQ were investigated to identify possible biases in response distributions. One approach was to examine the response mean for each item, centered on the mean of the Likert scale; we called this $M_{c t r}$. One would expect $M_{c t r}$ to approximate 0 for a normally distributed response pattern (i.e., no bias in either the positive or negative direction). Thus, $M_{c t r}$ was calculated for the BIMS and compared to the same statistics of equivalent items on the FFMQSF and FFMQ. Given potential response biases previously observed in some studies on the FFMQ (e.g., Van Dam et al., 2009), we believed that improvements to the way that questions were asked would result in a more normative distribution (i.e., less deviation from the scale center point). Analysis were conducted to further examine distributional bias and method effects as previously described (see e.g., Van Dam et al., 2012). We did this using both method effects as inspired by wording of FFMQ analogue items and based on BIMS item distributions.

\section{Results}

\section{Data Cleaning}

For the BIMS, the initial dataset consisted of $n=1,014$ cases. After removal of the cases that did not meet the criteria identified in the data cleaning plan, the final dataset was $N=$ 
767 (see Figure S1). The dataset was then randomly split into two datasets by allocating each participant a number using a random number generator and sorting the cases in ascending order by the random number. The first 450 cases were allocated to the EFA dataset and the remaining 316 cases to the CFA dataset.

\section{Descriptive Statistics}

The majority of the sample was male $(54.11 \%)$ with an average age of 36 years (SD =9.68). Most participants identified as White/Caucasian $(69 \% ; \mathrm{n}=529)$, a majority of the participants were never married $(45.9 \% ; n=352)$, had a College/University education or higher $(55.7 \% ; \mathrm{n}=427)$, and were employed full-time $(72.9 \% ; \mathrm{n}=559)$ (see Table S1 for more details).

\section{Exploratory Factor Analysis}

Parallel analysis suggested four factors. However, further examination of the scree plot showed that a $5^{\text {th }}$ factor was along the parallel analysis line (see Figure 1). The presence of a $5^{\text {th }}$ factor was plausible; the optimal number of factors was guided by both data and theory. Subsequently, both four- and five-factor models were examined. 


\section{Non Graphical Solutions to Scree Test}

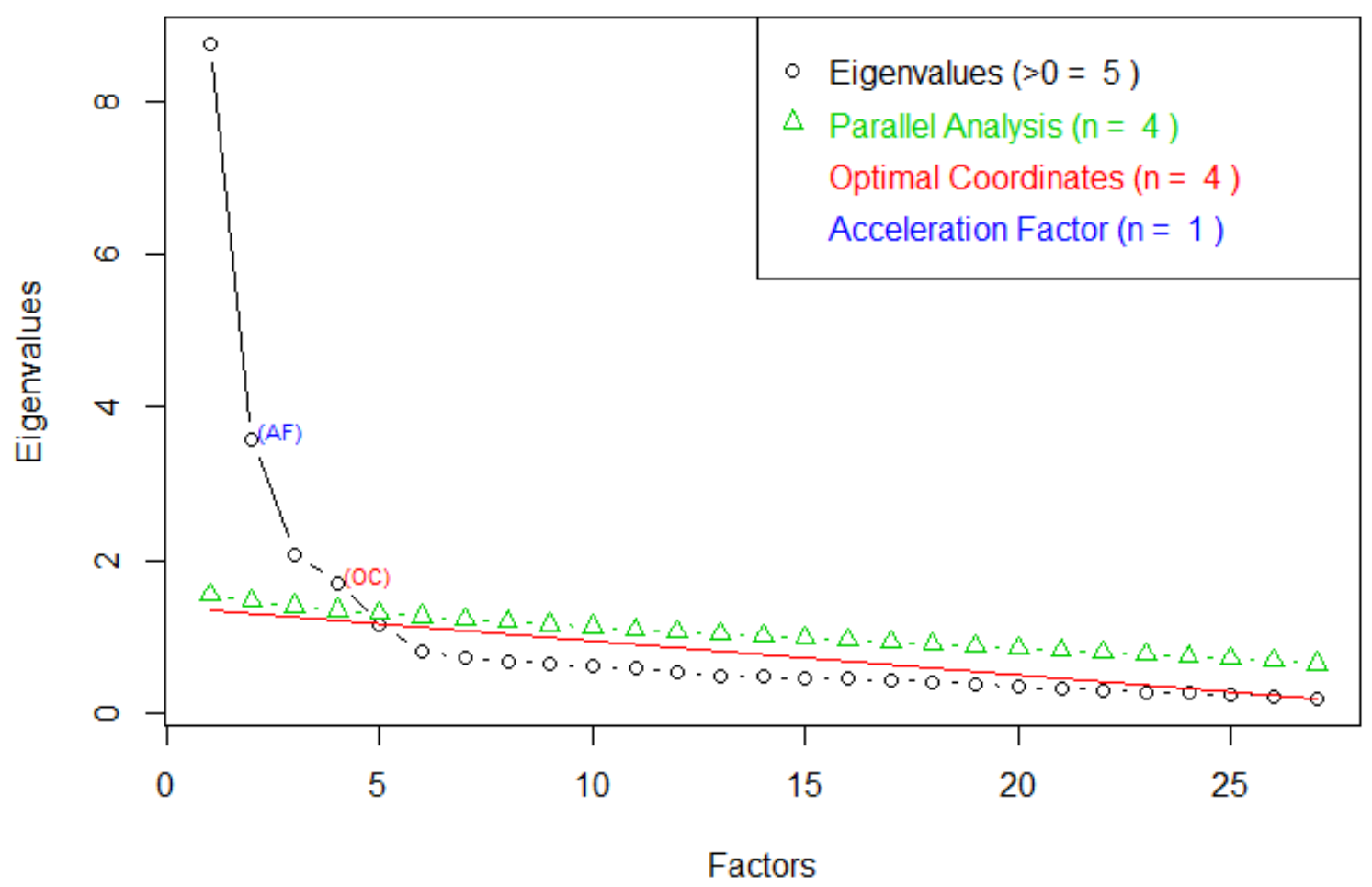

Figure 1. Scree plot with optimal coordinates at four factors and the parallel analysis line suggesting five factors.

Five-factor model. The pattern matrix of the original 30 item five-factor model (see

Table S2) showed issues for three items, which exhibited some cross-loading and did not align logically with the indicated factor (see Appendix A for BIMS items). All three items were thus deleted.

Four-factor model. The pattern matrix for the four-factor model suggested by the EFA generally combined the ActAware and NonReact items into a single factor. The other three factors (i.e., Observe, NonJudge, and Describe) followed the original FFMQ factor structure. Four cross-loadings items were identified and were deleted (i.e., bims1, bims19, bims24, and bims27). However, after deletion, the pattern matrix would not stabilize; more cross-loadings emerged and the items comprising the factors changed (now NonJudge and NonReact items 
represented a single factor). Deletion of more items would greatly have reduce the number of questions. As a result, $\mathrm{t}$ the 26 -item version would be the minimal number of items considered.

\section{Comparative Model Fit}

Comparative model fit indices for the five- and the four-factor models from the separate exploratory and confirmatory samples are shown in Table 2. The best model was the correlated five-factor model consisting of 27 items. CFA analyses were conducted separately (see Table 2). In both cases, the five-factor correlated 27-item model was the best model. To obtain the full estimates for the standardized factor loadings and model fit statistics, CFA analysis was also conducted on the full sample. For the 5F, correlated model, all criteria were consistent with good model-to-data fit. For the full sample, the standardized factor loadings for each item were strong across the factors; ranging $0.537-0.967$ (see Figure 2).

Table 2. Model fit statistics for EFA and CFA among corresponding samples.

\begin{tabular}{|c|c|c|c|c|c|c|c|c|c|}
\hline & $\begin{array}{l}\text { Factors } \\
\text { (Items) }\end{array}$ & Model & $\chi^{2}$ & $d f$ & CFI & TLI & RMSEA & $90 \% \mathrm{CI}$ & SRMR \\
\hline \multirow{4}{*}{$\begin{array}{c}\text { EFA } \\
n=450\end{array}$} & 5 Factor & Hier. & 851.39 & 319 & 0.967 & 0.963 & 0.061 & $0.056,0.066$ & 0.074 \\
\hline & (27) & Corr. & 343.19 & 314 & 0.998 & 0.998 & 0.014 & $0.000,0.024$ & 0.047 \\
\hline & \multirow{2}{*}{$\begin{array}{c}4 \text { Factor } \\
(26)\end{array}$} & Hier. & 841.35 & 295 & 0.962 & 0.958 & 0.064 & $0.059,0.069$ & 0.076 \\
\hline & & Corr. & 718.99 & 293 & 0.970 & 0.967 & 0.057 & $0.052,0.062$ & 0.070 \\
\hline \multirow{4}{*}{$\begin{array}{c}\text { CFA } \\
n=317\end{array}$} & \multirow{2}{*}{$\begin{array}{c}5 \text { Factor } \\
(27)\end{array}$} & Hier & 757.74 & 319 & 0.954 & 0.949 & 0.066 & $0.060,0.072$ & 0.082 \\
\hline & & Corr. & 339.39 & 314 & 0.997 & 0.997 & 0.016 & $0.000,0.027$ & 0.055 \\
\hline & \multirow{2}{*}{$\begin{array}{l}4 \text { Factor } \\
\text { (26) }\end{array}$} & Hier. & 1468.05 & 319 & 0.955 & 0.950 & 0.069 & $0.065,0.072$ & 0.074 \\
\hline & & Corr. & 538.73 & 314 & 0.991 & 0.990 & 0.031 & $0.026,0.035$ & 0.045 \\
\hline
\end{tabular}




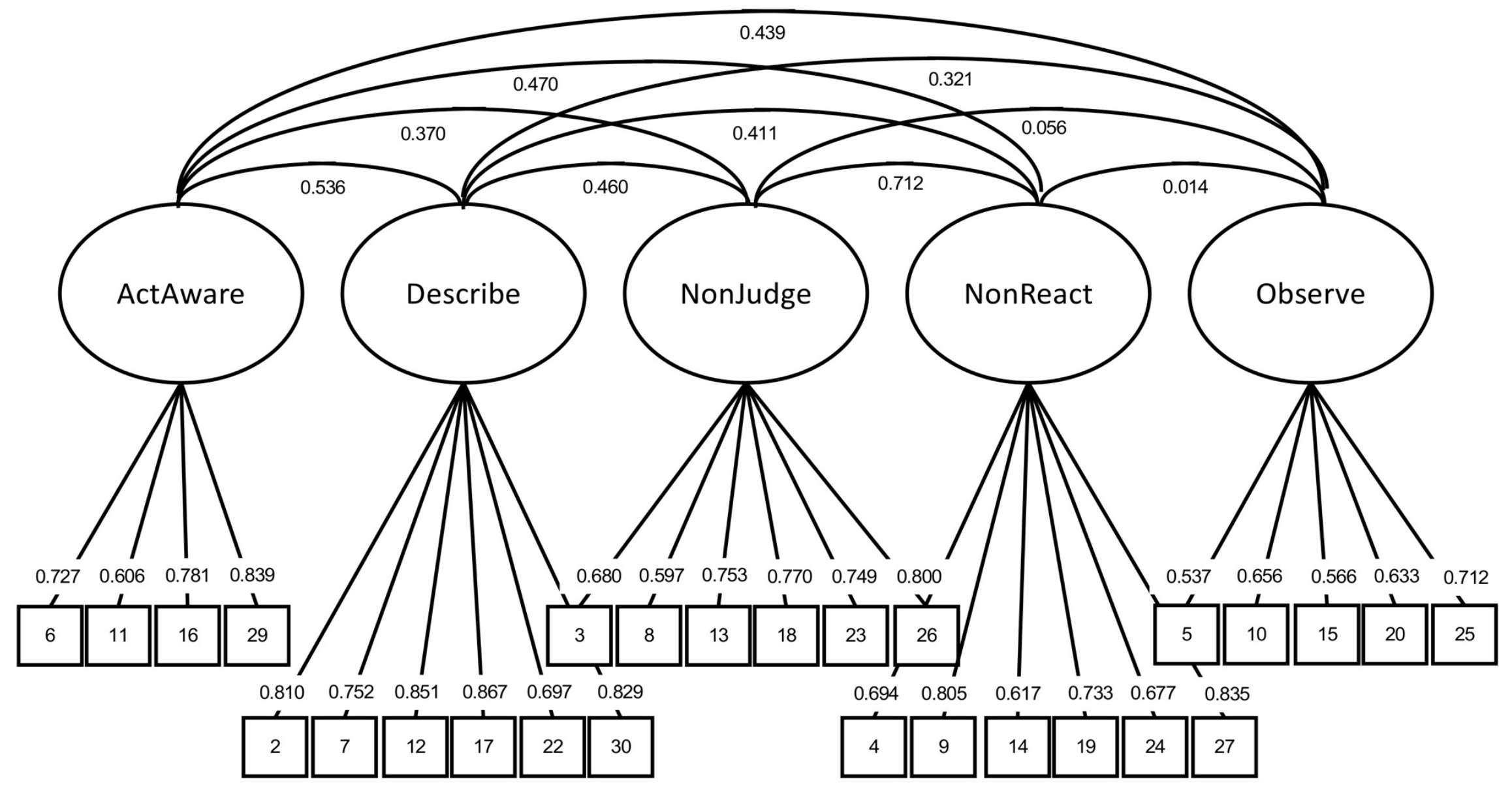

Figure 2. Factor structure, standardized factor loadings, and factor intercorrelations for full sample $(n=767)$ 


\section{Construct validity of the BIMS}

We explored correlations between the BIMS and FFMQ-SF to examine convergent validity. Subscales of the BIMS exhibited large correlations with the comparably named measures on the FFMQ-SF (ActAware: $r=0.77(95 \% \mathrm{CI}=0.68,0.75)$; Describe: $r=0.85(95 \%$ $\mathrm{CI}=0.83,0.87)$; NonJudge: $r=0.72(95 \% \mathrm{CI}=0.68,0.75)$; NonReact: $r=0.75(95 \% \mathrm{CI}=0.72$, 0.78); Observe: $r=0.75(95 \% \mathrm{CI}=0.72,0.78)$. Correlations between differently name subscales ranged from negligible values $(0.01)$ to moderately high associations $(0.50)$. Details of interscale correlations are provided in Table $\mathbf{S 4}$.

Correlations of the BIMS with other mindfulness-related scales were computed to determine if there were any differences in construct validity. The correlations of the BIMS and FFMQ-SF with other mindfulness-related scales were extremely similar. There were minimal differences: $85 \%$ of correlations exhibited absolute differences in $r \leq 0.1$ (see Table 6). There were a small number of correlations (6.3\%) that exhibited differences of $r \geq 0.1$. Most of these differences were observed among correlations with the ActAware and NonJudge subscales as they correlated to various subscales of the DERS. In all cases, the correlation was larger for the FFMQ-SF than the BIMS. 
Table 4. Correlations of BIMS \& FFMQ-SF with related measures $(N=767)$.

\begin{tabular}{|c|c|c|c|c|c|c|c|c|c|c|c|c|c|c|c|c|c|}
\hline \multirow[b]{2}{*}{ Scale } & \multirow[b]{2}{*}{ scale } & \multicolumn{3}{|c|}{ BIDR-SF } & \multirow[b]{2}{*}{ ACS } & \multicolumn{7}{|c|}{ DERS } & \multicolumn{5}{|c|}{ BFI } \\
\hline & & IM & SDE & Total & & $\mathbf{A}$ & $\mathbf{C}$ & $\mathbf{G}$ & I & $\mathbf{N A}$ & $\mathbf{S}$ & Total & $\mathbf{A}$ & $\mathrm{C}$ & $\mathbf{E}$ & $\mathbf{N}$ & $\mathbf{O}$ \\
\hline \multirow[t]{5}{*}{ BIMS } & $\mathbf{A A}$ & 0.33 & 0.42 & 0.44 & 0.40 & -0.32 & -0.40 & -0.32 & -0.35 & -0.32 & -0.35 & -0.45 & 0.27 & 0.50 & 0.25 & -0.35 & 0.30 \\
\hline & DE & 0.21 & 0.47 & 0.39 & 0.49 & -0.43 & -0.51 & -0.34 & -0.27 & -0.39 & -0.38 & -0.50 & 0.25 & 0.40 & 0.41 & -0.37 & 0.35 \\
\hline & $\mathbf{N J}$ & 0.29 & 0.54 & 0.48 & 0.38 & -0.12 & -0.30 & -0.38 & -0.34 & -0.56 & -0.47 & -0.49 & 0.20 & 0.34 & 0.27 & -0.52 & 0.12 \\
\hline & NR & 0.32 & 0.62 & 0.54 & 0.50 & 0.00 & -0.32 & -0.54 & -0.43 & -0.47 & -0.59 & -0.55 & 0.17 & 0.41 & 0.32 & -0.70 & 0.12 \\
\hline & $\mathbf{O}$ & 0.12 & 0.00 & 0.11 & 0.10 & -0.32 & -0.17 & 0.00 & 0.00 & 0.00 & 0.00 & -0.14 & 0.13 & 0.18 & 0.13 & 0.00 & 0.38 \\
\hline \multirow{5}{*}{$\begin{array}{l}\text { FFMQ } \\
\text {-SF }\end{array}$} & $\mathbf{A A}$ & 0.34 & 0.51 & 0.49 & 0.51 & -0.31 & -0.55 & -0.47 & -0.47 & -0.46 & -0.51 & -0.61 & 0.35 & 0.58 & 0.26 & -0.44 & 0.29 \\
\hline & DE & 0.20 & 0.48 & 0.39 & 0.51 & -0.44 & -0.54 & -0.36 & -0.31 & -0.42 & -0.42 & -0.54 & 0.26 & 0.40 & 0.39 & -0.38 & 0.38 \\
\hline & NJ & 0.29 & 0.47 & 0.44 & 0.35 & -0.12 & -0.40 & -0.39 & -0.44 & -0.67 & -0.51 & -0.57 & 0.26 & 0.32 & 0.19 & -0.46 & 0.15 \\
\hline & NR & 0.28 & 0.57 & 0.49 & 0.47 & -0.15 & -0.27 & -0.47 & -0.38 & -0.39 & -0.53 & -0.49 & 0.18 & 0.37 & 0.29 & -0.66 & 0.00 \\
\hline & O & 0.11 & 0.11 & 0.13 & 0.16 & -0.37 & -0.20 & 0.00 & -0.11 & 0.00 & 0.00 & -0.18 & 0.15 & 0.19 & 0.19 & 0.00 & 0.41 \\
\hline
\end{tabular}

Note. Values have been bolded where the difference in the correlation between BIMS and FFMQ-SF is $r \geq 0.1$.

BIMS = Balanced Inventory of Mindfulness-related Skills; FFMQ-SF = Five Facet Mindfulness Questionnaire,

Short Form; $\mathrm{AA}=$ ActAware DE = Describe; NJ = NonJudge; $\mathrm{NR}=$ NonReact $; \mathrm{O}=$ Observe; BIDR-SF $=$ Balanced

Inventory of Desirable Responding, Short Form; IM = Impression Management; SDE = Self Deceptive

Enhancement; ACS = Attentional Control Scale; DERS = Difficulties in Emotion Regulation Scale; A = Awareness;

$\mathrm{C}=$ Clarity $; \mathrm{G}=$ Goals $; \mathrm{I}=$ Impulse NA = NonAcceptance $; \mathrm{S}=$ Strategies $; \mathrm{BFI}=$ Big Five Inventory; $\mathrm{A}=$

Agreeableness $; \mathrm{C}=$ Conscientiousness $; \mathrm{E}=$ Extraversion; $\mathrm{N}=$ Neuroticism; $\mathrm{O}=$ Opennnes 


\section{Response Bias Mitigation}

The BIMS was similarly correlated with the BIDR-SF scales relative to the FFMQ-SF (see Table 6). For both the BIMS and FFMQ-SF, correlations with the Observe subscales and BIDR-SF subscales and total were negligible $(r<0.15)$. Interestingly, across all other subscales except Observe (for both BIMS and FFMQ-SF), correlations with the BIDR-SF were meaningfully higher with the Self-Deceptive Enhancement subscale $(r$ 's $\approx 0.4-0.6)$ than the Impression Management subscale $\left(r^{\prime} \mathrm{s} \approx 0.2-0.35\right)$. Item means for the BIMS were also investigated to identify possible biases in response distributions. Considering the statistic (i.e., $M_{c t r}$ ) estimated to represent potential item-level bias (see Table 7), the BIMS exhibited less extreme deviation from the mean $(M=0.34)$ relative to the FFMQ-SF $(M=0.44)$ and FFMQ $(M=0.56)$. 
Table 5. Comparison of item properties for comparable items on BIMS, FFMQ-SF, and FFMQ.

\begin{tabular}{|c|c|c|c|c|c|c|}
\hline Subscale & BIMS & $M_{c t r}$ & FFMQ-SF & $M_{c t r}$ & FFMQ & $M_{c t r}$ \\
\hline \multirow[t]{4}{*}{ ActAware } & 6 & 0.22 & $12^{\mathrm{R}}$ & -0.49 & $23^{\mathrm{R}}$ & -0.5 \\
\hline & 11 & 0.74 & $17^{\mathrm{R}}$ & -0.77 & $28^{\mathrm{R}}$ & -0.4 \\
\hline & 16 & 0.81 & $23^{\mathrm{R}}$ & -0.74 & $38^{\mathrm{R}}$ & -0.17 \\
\hline & 29 & 0.86 & $22^{\mathrm{R}}$ & -0.63 & $34^{\mathrm{R}}$ & -0.54 \\
\hline \multirow[t]{6}{*}{ Describe } & 2 & 0.36 & 1 & 0.5 & 2 & 0.75 \\
\hline & 7 & 0.69 & 2 & 0.64 & 7 & 0.85 \\
\hline & 12 & 0.48 & $5^{\mathrm{R}}$ & -0.53 & $12^{\mathrm{R}}$ & -0.84 \\
\hline & 17 & 0.34 & 16 & 0.31 & 27 & 0.61 \\
\hline & 22 & 0.27 & N/A & N/A & 32 & 0.7 \\
\hline & 30 & 0.5 & $11^{\mathrm{R}}$ & -0.45 & $22^{\mathrm{R}}$ & -0.86 \\
\hline \multirow[t]{6}{*}{ NonJudge } & 3 & -0.09 & $4^{\mathrm{R}}$ & -0.32 & $10^{\mathrm{R}}$ & -0.68 \\
\hline & 8 & -0.13 & $7^{\mathrm{R}}$ & -0.03 & $17^{\mathrm{R}}$ & -0.29 \\
\hline & 13 & -0.02 & $14^{\mathrm{R}}$ & -0.39 & $25^{\mathrm{R}}$ & -0.76 \\
\hline & 18 & 0.45 & $19^{\mathrm{R}}$ & -0.46 & $30^{\mathrm{R}}$ & -0.79 \\
\hline & 23 & -0.22 & $24^{\mathrm{R}}$ & -0.38 & $39^{\mathrm{R}}$ & -0.82 \\
\hline & 26 & 0.51 & N/A & $\mathrm{N} / \mathrm{A}$ & $35^{\mathrm{R}}$ & -0.45 \\
\hline \multirow[t]{6}{*}{ NonReact } & 4 & 0 & 3 & 0.41 & 9 & 0.27 \\
\hline & 9 & 0.33 & 9 & 0.44 & 19 & 0.12 \\
\hline & 14 & 0.08 & N/A & N/A & 21 & 0.35 \\
\hline & 19 & 0.12 & 13 & 0.16 & 24 & 0.3 \\
\hline & 24 & 0.08 & 18 & 0.15 & 29 & -0.03 \\
\hline & 27 & 0.09 & 21 & 0.11 & 33 & -0.16 \\
\hline \multirow[t]{5}{*}{ Observe } & 5 & -0.18 & N/A & N/A & 1 & 0.08 \\
\hline & 10 & 0.32 & 6 & 0.51 & 15 & 0.9 \\
\hline & 15 & 0.36 & 10 & 0.41 & 20 & 0.82 \\
\hline & 20 & 0.54 & 15 & 0.72 & 26 & 1.02 \\
\hline & 25 & 0.44 & 20 & 0.64 & 31 & 0.98 \\
\hline $\mid$ Mean $\mid$ & & 0.34 & & 0.44 & & 0.56 \\
\hline $\mid$ Median $\mid$ & & 0.33 & & 0.45 & & 0.61 \\
\hline
\end{tabular}

Note: Values were calculated prior to any reverse-scoring. $M_{c t r}$ indicates item mean minus scale mean (i.e., BIMS $=3.5$, FFMQ-SF/FFMQ $=3$ ); ${ }^{\mathrm{R}}$ Indicates item is intended to be reverse-coded. Color intensity based on numeric number ranging from $\approx-1$ (red) to 1 (green); BIMS $=$ Balanced Inventory of Mindfulness-related Skills; FFMQ = Five Facet Mindfulness Questionnaire; - SF = Short Form. 
Multiple method effects models were explored to ascertain mitigation of method effects on the FFMQ (see Van Dam et al 2012) via revisions implemented to develop the BIMS. Modelto-data fits were compared to fits for the traditional models (5 Factor Hierarchical and 5 Factor Correlational). We explored FFMQ-item inspired method effects models (i.e., ascertaining positive or negative method loading based on wording direction of original FFMQ item) and BIMS-specific direction effects (i.e., using item level distribution information). Across all 6 method effects models, no method effects models provided even adequate fit to the data (see

Table S5). All CFI/TLI values were below 0.900, only one model exhibited a RMSEA and SRMR below 0.10 (Negative Method Model per FFMQ).

\section{Discussion}

The goal of this study was to examine whether revision of items from the FFMQ, presented in the structured alternative format using a 1 to 6 even-numbered response format would improve psychometric limitations previously observed on the FFMQ while maintaining construct validity. A 30-item scale, reduced during analysis to 27 items, representing five correlated factors (four items for ActAware, six items for Describe, six items for NonJudge, six items for NonReact, five items for Observe) provided excellent model-to-data fit, consistent with the proposed model for the FFMQ. The correlated model still fit the data better than a hierarchical model, consistent with prior observations of the FFMQ (see e.g., Karl et al., 2020; Van Dam et al., 2012) and with original recommendations by the scale developers (Baer et al. 2006). The fact that a correlated model was most robust implies a set of related skills rather than constructs that add to a single entity that could be characterized as Mindfulness. The subscales of the BIMS correlated strongly with their FFMQ-SF counterparts (e.g., $r>0.72$ ) and correlations among subscales and measures of attentional control, emotion regulation, and big- 5 personality traits were largely comparable between the BIMS and FFMQ-SF. Contrary to predictions, the BIMS did not exhibit smaller correlations with measures of socially desirable responding 
(especially self-deceptive enhancement). It is worth noting that the BIMS did exhibit a more normative distribution in responses than did the FFMQ, with the average response falling closer to the mean more often than was the case for comparable data on the FFMQ. Additionally, no method effects models provided even adequate model-to-data fit for the BIMS (in contrast to the FFMQ-SF), suggesting that the new scale achieved one part of the goals (i.e., eliminating potential method effects). By way of comparison, the FFMQ-SF mitigated psychometric issues previously observed in the full scale (see Van Dam et al. 2012) but still exhibited negative method effects and was not best characterized as measuring a single higher-order construct but rather a collection of constructs.

\section{Factor Structure}

Parallel analysis indicated that a possible 4- or 5-factor structure to the BIMS. Comparing different models, a correlated 5-factor model exhibited the best model-to-data fit, exceeding standards for excellent model fit (CFI \& TLI $>0.95$, RMSEA $<.06$, SRMR $<.08$; Hu \& Bentler, 1999). Final structure of the data mirrored that of the FFMQ and after item examination, the scale was reduced from 30 to 27 items.

The fact that a correlated (or group-factor) rather than hierarchical (or second-order factor) model best fits the data has interesting implications for how to think about scale and subscale scores (see Rindskopf \& Rose, 1988). The BIMS, as well as the FFMQ-SF (and based on prior data, the FFMQ; see e.g., Van Dam et al., 2012), is seemingly not best explained by a second-order latent variable that is a combination of the first-order latent variables (i.e., ActAware, Describe, NonJudge, NonReact, Observe). The suggestion that the FFMQ and related measures reflect potentially associated skills rather a single, agglomerative construct of mindfulness is consistent with recommendations by Baer and colleagues (2006). The scale would seem to represent five related latent variables that exhibit modest correlations with one another. Given that recent work has suggested that the subscales of the FFMQ may not be viable on their 
own (see Pelham III et al., 2019), it would seem that the scale should be administered as a single entity, using the subscales as informative units. In other words, the present analyses suggest that using a total sum score, combining all 27 items, is ill-advised. We would recommend that the BIMS be considered as a collection of related skills rather than as a single, unitary measure of mindfulness. It is also worth noting that some of the most useful patterns may be identified when considering interactions among these skillsets (see e.g., Eisenlohr-Moul et al., 2012). The idea of measuring potentially related skills may also mitigate concerns about whether or not such scales can measure complex concepts such as Mindfulness ( Grossman \& Van Dam, 2011; Baer 2019).

We would like to note that we did not consider a Bifactor model in examining the present data, though we did implement such a model in our preliminary work (Van Dam, Bilgrami, \& Eisenlohr-Moul, 2018). Despite an increasing resurgence of fitting bifactor models recently (Markon, 2019; Reise, 2012), it is worth noting that there are important limitations to the bifactor model. Among comparable, nested models (i.e., one-factor model, second-order factor model or hierarchical model, group-factor or correlated factors model, bifactor model) of the same data, a bifactor model is the least restricted model (Rindskopf \& Rose, 1988) and therefore most likely to suggest evidence of good model-to-data fit under nearly all circumstances (see e.g., Reise et al., 2016). Since bifactor models tend to overfit data, it is critical that one considers whether the model is actually a plausible explanation for the data and the scale that was used to collect the data. In the present case, and similar cases, we contend that there is not a general factor that best represents mindfulness independently of specific factors (e.g., factors related to the subscales). In fact, the present data would suggest that a second-order factor may not even be a good representation of how these skills fit together. Moreover, it's possible that the skills do not fit together in trait-like ways but rather interact dynamically to contribute to mindful states.

\section{Convergent Validity}


The BIMS subscales were highly correlated to their counterparts on the FFMQ-SF with > $50 \%$ shared variance among them. The BIMS scales exhibited medium-sized correlations among each other, with the exception of the Observe scale, which exhibited no relationship with NonJudge and NonReact. The lack of correlation of the Observe scale with NonJudge and Nonreact is not surprising given negligible correlations in the original development (Baer et al., 2006), as well as known differences in how the Observe scale behaves among meditators and non-meditators (Baer et al., 2008).

The pattern of correlations of BIMS and FFMQ-SF with attentional control, emotion regulation, and big-5 personality traits were comparable to one another. There were only differences between BIMS and FFMQ-SF of $|r|>0.1$ among eight of 80 correlations (10\%). Six of these eight differences pertained to the ActAware scale. In all cases, the correlation between ActAware and other measures decreased from the FFMQ-SF to the BIMS. As the ActAware subscale in the FFMQ is largely comprised of items from the MAAS, and the MAAS exhibits known method effects (see e.g., Höfling et al., 2011), it is plausible that these changes are the result of a reduction in method effects. Notably, method effects due to reverse-wording on the DERS have also been identified in prior psychometric works of that measure (see e.g., Bardeen et al. 2016).

Correlations of the BIMS and FFMQ-SF with attentional control were largely comparable, with the main difference being related to the ActAware scale (as discussed above). Correlations of the BIMS and FFMQ-SF with emotion regulation were largely comparable as well, with meaningful correlations (i.e., $|r| \geq 0.25$ ) among 32 of 40 evaluated potential associations. The most consistent differences were among ActAware, with five of the six subscales, as well as the total score of the DERS, wherein the BIMS correlation with the DERS was reliably smaller than the FFMQ-SF correlation with the DERS. There were also two correlations, among NonJudge (with emotional clarity and impulse) that were smaller on the 
BIMS than the FFMQ-SF. Correlations with the DERS total score were comparable to those of the original scale, with only the Observe subscale not exhibiting a meaningful relationship (see Baer et al., 2006). The continued overlap of the BIMS (similar to the FFMQ) with emotion regulation may represent meaningful association or construct overlap (see Roemer et al., 2015). As mindfulness-related skills are conceptualized as emotion regulation behaviors in certain contemporary psychotherapies (e.g., Dialectical Behavior Therapy), this overlap is logical. More work is needed to ascertain whether self-reported emotion regulation can be adequately distinguished from self-reported mindfulness. It may be that these constructs fundamentally overlap in terms of the way that we ask about them.

With respect to correlations among the subscales and personality traits, the correlations were not only comparable to one another but were also highly consistent with a recently metaanalytic review (Hanley \& Garland, 2017). For each subscale, the most notable correlations were ActAware with Conscientiousness, Describe with Conscientiousness and Extraversion, NonJudge with Neuroticism, NonReact with Neuroticism, and Observe with Openness. Interestingly, the BIMS correlations with Personality exhibited slightly more specificity than the FFMQ-SF or FFMQ (see Hanley \& Garland, 2017).

\section{Divergent Validity - Relationship to Social Desirability}

Contrary to expectations, the BIMS did not exhibit reduced correlations with desirable responding relative to the FFMQ-SF. Although the potential influence of social desirability has been postulated (Grossman, 2008), and some have controlled or social desirability in regression models (see e.g., Jensen et al., 2019), few studies have directly examined relationships between self-reported mindfulness and social desirability. There were no meaningful differences among the BIMS or FFMQ-SF in relation to the BIDR-SF. For both the BIMS and FFMQ-SF, the Observe subscale was not correlated to desirable responding. All other subscales, however, were associated with impression management (except Describe), self-deceptive enhancement, and 
total social desirability. Excluding Observe, across both the BIMS and FFMQ-SF, correlations with social desirability were stronger for self-deceptive enhancement $(r$ 's $=0.42-0.62)$ than impression management ( $r$ 's $=.20-.34)$. Self-deceptive enhancement refers to a nonconscious inclination to be positive about oneself while impression management is a more conscious inflation of self-descriptions (see Hart et al., 2015). Notably, it is not necessarily a problem that one is (overly) optimistic about one's capacities (as indicated by elevated SDE scores), though elevation here may conflate aspiration with achievement. Grossman (2008) has suggested exactly this possibility; perhaps individuals are endorsing mindfulness items because they want to be more mindful, not necessarily because they are more mindful (though admittedly, it could be a combination of both). The revision of the items in the BIMS (from the FFMQ) did not mitigate such desirability and it will be important to explore this issue further, especially within the context of mindfulness-based practice.

\section{Response Distributions and Method Effects}

Examining response distributions on the BIMS and data from the same items on the FFMQ-SF and FFMQ (taken from Pang \& Ruch, 2019), BIMS items were, on average, closer to the mean, suggesting we successfully reduced bias related to wording effects. Thus, it may be that the BIMS better reflects normally distributed characteristics related to mindfulness than the FFMQ. When examining method effects models on the BIMS, no methods effect models provided adequate model-to-data fit, suggesting that the revisions undertaken to develop the BIMS eliminated the method effects associated with the FFMQ. It is interesting to note that while method effects were essentially eliminated in the BIMS, associations with social desirability were not. These results suggest that method effects in the assessment of mindfulnessrelated constructs may be, at least partially, independent of social desirability. Further work will be required to explore the extent to which social desirability influences responses on mindfulness measures more broadly. 


\section{Broader Measurement Issues}

It is important to note that we believe that the BIMS reflects skills related to mindfulness and not necessarily mindfulness, itself. Our thinking regarding mindfulness-related skills harkens to the KIMS (Baer et al. 2004), which proposed to assess a particular set of skills associated with a particular therapeutic orientation (i.e., Dialectical Behavior Therapy). It is unclear whether a construct such as mindfulness (especially as reflected in traditional Buddhist contexts) can actually be evaluated via self-report (see e.g., Grossman \& Van Dam, 2011). The development of the BIMS was not a proposal to resolve this important question of content validity but rather an effort to improve one of the most widely used scales (that exhibits considerable clinical utility) while also shifting the conversation back to skillsets rather than traits. The FFMQ has recently been shown to lack metric equivalence across 16 different countries (Karl et al. 2020). We suspect that the BIMS may exhibit similar problems with respect to how different cultures understand and interpret the items. It may very well be that conceptualizations of the skills related to mindfulness practice vary considerably as a function of whether one has pre-existing knowledge of Buddhist ideas and/or a history of meditation practice. We do not contend that the BIMS is a one measure to rule them all but rather a way towards measuring mindfulness-related skills as they are learned and taught in contemporary western psychotherapies. It would seem that these skills are also pertinent to certain mindfulness-based interventions but may not be pertinent to certain mindfulness and/or meditation practices more generally.

\section{Limitations and Future Research}

It is important to note that there are a number of limitations associated with the present work. The most notable limitation is that we collected data in a convenience sample and did not measure meditation history. It is very possible that meditation history alters the way that individuals interpret the items of the BIMS and the psychometric properties of the scale (see e.g., Baer et al. 2008; Van Dam et al. 2009). An additional limitation is that we did not collect data 
before and after a gold-standard Mindfulness-based Intervention. As such, we do not know whether the scale tracks with changes specific to mindfulness or if it may, similar to the FFMQ (Goldberg et al. 2016), merely track with broader psychological changes. It is also possible that it does not reflect changes in mindfulness at all, again similar to the FFMQ (Hsiao et al. 2019). It is important to note, however, that the content for the BIMS is fundamentally derived from the FFMQ and the goal was not to validate a measure of 'mindfulness' but to improve the psychometric limitations of the FFMQ. As the present scale tracks closely with the FFMQ, it is likely that it has similar measurement properties in relationship to MBIs to the FFMQ. Further work is required to explore this possibility.

The present work represents what we believe to be an important advance in the way that mindfulness is measured with a shift towards improved psychometric properties and away from the idea that mindfulness can be assessed in an agglomerative fashion. The steps taken to develop the BIMS eliminate the method effects of its predecessor (i.e., FFMQ). Further, the BIMS is highly correlated with its predecessor, shows good convergent validity, and retains the five-factor structure of the original scale. While aspects of social desirability were not eliminated, it may be that disentangling aspiration from achievement requires formal assessment as has been done with other measures (see e.g., Tsai, 2007). We believe that the BIMS is an advance in the assessment of mindfulness-related skills and could herald an important shift in how we measure the construct (in a balanced way to mitigate desirability) and how we think about it (as a collection of skills rather than a unidimensional trait). 


\section{Ethics Statement}

All work contained within the present manuscript have been approved by the University of Melbourne ethics committee and therefore have been performed in accordance with the ethical standards laid down in the 1964 Declaration of Helsinki and its later amendments.

\section{Author Contributions}

SSP: helped design and execute the study, assisted with the data analyses, and wrote the first draft of the paper. ZRB: collaborated with the design and writing of the study. TE: collaborated in the design, as well as writing and editing of the final manuscript. NTVD: oversaw design and execution of the study, implemented or oversaw data analyses, and revised the manuscript. All authors approved the final version of the manuscript for submission. 


\section{References}

Baer, R. (2019). Assessment of mindfulness by self-report. Current Opinion in Psychology, 28, 42-48. doi:10.1016/j.copsyc.2018.10.015

Baer, R. A. (2011). Measuring mindfulness. Contemporary Buddhism, 12(1), 241-261. doi:10.1080/14639947.2011.564842

Baer, R. A., Smith, G. T., \& Allen, K. B. (2004). Assessment of mindfulness by self-report: The Kentucky Inventory of Mindfulness Skills. Assessment, 11(3), 191-206. doi:10.1177/1073191104268029

Baer, R. A., Smith, G.T., Hopkins, J., Krietemeyer, J., \& Toney, L. (2006). Using self-report assessment method to explore facets of mindfulness. Assessment, 13(1), 27-45. doi:10.1177/1073191105283504

Baer, R. A., Smith, G. T., Lykins, E., Button, D., Krietemeyer, J., Sauer, S., ...Williams, J. M. G. (2008). Construct validity of the Five Facet Mindfulness Questionnaire in meditating and nonmeditating samples. Assessment, 15(3), 329-342. doi:10.1177/1073191107313003

Bardeen, J.R., Fergus, T.A., Hannan, S.M., \& Orcutt, H.K. (2016). Addressing psychometric limitations of the Difficulties in Emotion Regulation Scale through item modification. Journal of Personality Assessment, 98, 298-309. doi:10.1080/00223891.2015.1091774

Barnhofer, T., Duggan, D. S., \& Griffith, J. W. (2011). Dispositional mindfulness moderates the relation between neuroticism and depressive symptoms. Personality and Individual Differences, 51(8), 958-962. doi:10.1016/j.paid.2011.07.032 
Baumeister, R. F., Vohs, K. D., \& Funder, D. C. (2007). Psychology as the science of selfreports and finger movements: Whatever happened to actual behavior? Perspectives on Psychological Science, 2(4), 396-403. doi:10.1111/j.1745-6916.2007.00051.x

Bohlmeijer, E., ten Klooster, P. M., Fledderus, M., Veehof, M., \& Baer, R. (2011). Psychometric properties of the Five Facet Mindfulness Questionnaire in depressed adults and development of a short form. Assessment, 18(3), 308-320. doi:10.1177/1073191111408231

Brown, K. W., \& Ryan, R. M. (2003). The benefits of being present: Mindfulness and its role in psychological well-being. Journal of Personality and Social Psychology, 84(4), 822-848. doi:10.1037/0022-3514.84.4.822

Campbell, D. T., \& Fiske, D. W. (1959). Convergent and discriminant validation by the multitrait-multimethod matrix. Psychological Bulletin, 56, 81-105. doi: $10.1037 / \mathrm{h} 0046016$

Christopher, M. S., Christopher, V., \& Charoensuk, S. (2009). Assessing “Western” mindfulness among Thai Theravāda Buddhist monks. Mental Health, Religion \& Culture, 12(3), 303314. doi:10.1080/13674670802651487

Christopher, M. S., Neuser, N. J., Michael, P. G., \& Baitmangalkar, A. (2012). Exploring the psychometric properties of the Five Facet Mindfulness Questionnaire. Mindfulness, 3, 124-131. doi:10.1007/s12671-011-0086-X

Cohen, J. (1992). A power primer. Psychological Bulletin, 112(1), 155-159. doi:10.1037/00332909.112.1.155

Conners, C.K., Erhardt, D., Sparrow, E. (1999). Conners' Adult ADHD Rating Scales: Technical Manual. North Tonawanda, NY: Multi-Health Systems, Inc. 
Costello, J.W., \& Osborne, J. W. (2005). Best practices in exploratory factor analysis: Four recommendations for getting the most from your analysis. Practical Assessment, Research \& Evaluation, 10(7), 1-9. doi:10.7275/jyj1-4868

de Bruin, E. I., Topper, M., Muskens, J. G. A. M., Bögels, S. M., \& Kamphuis, J. H. (2012). Psychometric properties of the Five Facets Mindfulness Questionnaire (FFMQ) in meditating and non-meditating samples. Assessment, 19(2), 187-197. doi:10.1177/1073191112446654

Derryberry, D., \& Reed, M. A. (2002). Anxiety-related attentional biases and their regulation by attentional control. Journal of Abnormal Psychology, 111(2), 225-236. doi:10.1037/0021843X.111.2.225

DiStefano, C., \& Motl, R. W. (2006). Further investigating method effects associated with negatively worded items on self-report surveys. Structural Equation Modeling, 13(3), 440-464. doi:10.1207/s15328007sem1303_6

Drasgow, F. \& Lissak, R. (1983). Modified parallel analysis: A procedure for examining the latent dimensionality of dichotomously scored item responses. Journal of Applied Psychology, 68, 363-363.

Dunn, A. M., Heggestad, E. D., Shanock, L. R., \& Theilgard, N. (2008). Intra-individual response variability as an indicator of insufficient effort responding: comparison to other indicators and relationships with individual differences. Journal of Business and Psychology, 33, 105-121. doi:10.1007/s10869-016-9479-0

Eisenlohr-Moul, T. A., Walsh, E. C., Charnigo, R. J., Lynam, D. R., \& Baer, R. A. (2012). The "What" and "How" of dispositional mindfulness: Using interactions among subscales of the Five-Facet mindfulness Questionnaire to understand its relation to substance use. Assessment, 19(3), 276-286. doi:10.1177/1073191112446658 
Enkavi, A. Z., Eisenberg, I. W., Bissett, P. G., Mazza, G. L., MacKinnon, D. P., Marsch, L. A., \& Poldrack, R. A. (2019). Large-scale analysis of test-retest reliabilities of self-regulation measures. Proceedings of the National Academy of Sciences of the United States of America, 116(12), 5472-5477. doi:10.1073/pnas.1818430116

Giluk, T. L. (2009). Mindfulness, big five personality, and affect: A meta-analysis. Personality and Individual Differences, 47, 805-811. doi: 10.1016/j.paid.2009.06.026

Goldberg, S. B., Wielgosz, J., Dhal, C., Schuyler, B., MacCoon, D., S., Rosenkranz, M., ... Davidson, R. J. (2016). Does the Five facet Mindfulness Questionnaire measure what we think it does? Construct validity evidence from an active controlled randomized clinical trial. Psychological Assessment, 28(8), 1009-1014. doi:10.1037/pas0000233

Grossman, P. (2008). On measuring mindfulness in psychosomatic and psychological research. Journal of Psychosomatic Research, 64(4), 405-408. doi:10.1016/j.jpsychores.2008.02.001

Grossman, P., \& Van Dam, N. T. (2011). Mindfulness, by any other name...: Trials and tribulations of Sati in western psychology and science. Contemporary Buddhism, 12(1), 219-239. doi:10.1080/14639947.2011.564841

Gu, J., Strauss, C., Crane, C., Barnhofer, T., Karl, A., Cavanagh, K., \& Kyuken, W. (2016). Examining the factor structure of the 39-item and 15-item versions of the Five Facet Mindfulness Questionnaire before and after mindfulness-based cognitive therapy for people with recurrent depression. Psychological Assessment, 28(7), 791-802. doi:10.1037/pas0000263

Hanley, A. W., \& Garland, E. L. (2017). The mindful personality: A meta-analysis from a cybernetic perspective. Mindfulness, 8(6), 1456-1470. doi:10.1007/s12671-017-0736-8 
Hart, C. M., Ritchie, T. D., Hepper, E. G., \& Gebauer, J. E. (2015). The Balanced Inventory of Desirable Responding short form (BIDR-16). SAGE Open, 5(4), 1-9. doi:10.1177/2158244015621113

Harter, S., \& Messer, B. (2012). The self-perception profile for adults: Manual and questionnaires. Denver: University of Denver.

Höfling, V., Moosbrugger, H., Schermelleh-Engel, K., \& Heidenreich, T. (2011). Mindfulness or mindlessness? A modified version of the Mindful Attention and Awareness Scale (MAAS). European Journal of Psychological Assessment, 27(1), 59-64. doi:10.1027/1015-5759/a000045

Hölzel, B. K., Lazar, S. W., Gard, T., Schuman-Olivier, Z., Vago, D. R., \& Ott, U. (2011). How does mindfulness meditation work? Proposing mechanisms of action from a conceptual and neural perspective. Perspectives on Psychological Science, 6, 537-559. doi: $10.1177 / 1745691611419671$

Horn, J. L. (1965). A rationale and test for the number of factors in factor analysis. Psychometrika, 30(2), 179-185. doi:10.1007/BF02289447

Hsiao, Y-Y, Tofiqhi, D., Keuger, E.S., Lee Van Horn, M., MacKinnon, D. P., \& Witkiewitz, K. (2019). The (lack of) replication of self-reported mindfulness as a mechanism of change in mindfulness-based relapse prevention for substance use disorders. Mindfulness, 10(4), 724-736. doi:10.1007/s12671-018-1023-Z

Hu, L., \& Bentler, P. M. (1999). Cutoff criteria for fit indexes in covariance structure analysis: Conventional criteria versus new alternatives. Structural Equation Modeling, 6(1), 1-55. doi:10.1080/10705519909540118 
Jensen, C. G., Krogh, S. C., Westphael, G., \& Hjordt, L.V. (2019). Mindfulness is positively related to socioeconomic jo status and income and independently predicts mental distress in a long-term perspective: Danish validation studies of the Five-Factor Mindfulness Questionnaire. Psychological Assessment, 31(1), e1-e20. doi:10.1037/pas0000667

Johnson, J. A. (2005). Ascertaining the validity of individual protocols from Web-based personality inventories. Journal of Research in Personality, 39, 103-129. doi:10.1016/j.jrp.2004.09.009

Kabat-Zinn, J. (2013). Full catastrophe living: How to cope with stress, pain and illness using mindfulness meditation (Rev. ed.). London: Piatkus.

Kalmet, N. \& Fouladi, R. T. (2008). A comparison of Physical Self-Perception Profile questionnaire formats: Structured alternative and ordered response scale formats. Measurement in Physical Education and Exercise Science, 12, 88-112. doi.org/10.1080/10913670801904025

Karl, J. A., Prado, S. M. M., Gračanin, A., Verhaeghen, P., Ramos, A., Mandal, S. P., ...Fischer, R. (2020). The cross-cultural validity of the Five-Facet Mindfulness Questionnaire across 16 countries. Mindfulness. doi:10.1007/s12671-020-01333-6

Kaufman, E. A., Xia, M., Fosco, G., Yaptangco, M., Skidmore, C. R., \& Crowell, S. E. (2016). The Difficulties of Emotion Regulation scale short form (DERS-SF): Validation and replication in adolescent and adult samples. Journal of Psychopathology and Behavioural Assessment, 38(3), 443-455. doi:10.1007/s10862-015-9529-3

Kulas, J. T., \& Stachowski, A. A. (2009). Middle category endorsement in odd-numbered Likert response scales: Associated item characteristics, cognitive demands, and preferred meanings. Journal of Research in Personality, 43, 489-493. doi:10.1016/j.jrp.2008.12.005 
Kulas, J. T., Stachowski, A. A., \& Haynes, B. A. (2008). Middle response functioning in Likertresponses to personality items. Journal of Business \& Psychology, 22(3), 251-259. doi:10.1007/s10869-008-9064-2

Lantz, B. (2013). The large sample size fallacy. Scandinavian Journal of Caring Sciences, 27, 487-492. doi:10.1111/j.1471-6712.2012.01052.x

Lecuona, O., García-Garzón, E., García-Rubio, C., \& Rodríguez-Carvajal, R. (2019). A psychometric review and conceptual replication study of the Five Facets Mindfulness Questionnaire latent structure. Assessment. doi:10.1177/1073191119873718

Li, C-H. (2016). Confirmatory factor analysis with ordinal data: Comparing robust maximum likelihood and diagonally weighted least squares. Behavioural Research Methods, 48(3), 936-949. doi:10.3758/s13428-015-0619-7

Lilja, J. L., Frodi-Lundgren, A., Hanse, J. J., Josefsson, T., Lumdh, L-G, Sköld, C., ... Broberg, A. G. (2011). Five Facets Mindfulness Questionnaire - reliability and factor structure: A Swedish Version. Cognitive Behaviour Therapy, 40(4), 291-303. doi:10.1080/16506073.2011.580367

Markon, K. E. (2019). Bifactor and hierarchical models: Specification, inference, and interpretation. Annual Review of Clinical Psychology, 15, 51-69. doi:10.1146/annurevclinpsy-050718-095522

Medvedev, O. N., Siegert, R. J., Kersten, P., \& Krägeloh, C. U. (2017). Improving the precision of the Five Facet Mindfulness Questionnaire using a Rash approach. Mindfulness, 8, 9951008. doi:10.1007/s12671-016-0676-8

Michalak, J., Zarbock, G., Drews, M., Otto, D., Mertens, D., Ströhle, G., ... Heidenreich, T. (2016). Erfassung von Achtsamkeit mit der deutschen Version des Five Facet 
Mindfulness Questionnaires (FFMQ-D). Zeitschrift für Gesundheitpsychologie, 24, 1-12. doi:10.1026/0943-8149/a000149

Morgan, T. A., Dalrymple, K., Multach, M., \& Zimmerman, M. (2017). Relations between mindfulness processes, psychiatric symptoms, and functioning in psychiatric and prebariatric surgery outpatients. Mindfulness, 8, 417-432. doi:10.1007/s12671-016-0614-9

Pang, D., \& Ruch, W. (2019). Scrutinizing the components of mindfulness: Insights from current, past, and non-meditators. Mindfulness, 10, 492-505. doi:10.1007/s12671-0180990-4

Pelham III, W. E., Gonzalez, O., Metcalf, S. A., Whicker, C. L., Scherer, E. A., Witkiewitz, K., ... Mackinnon, D. P. (2019). Item response theory analysis of the Five Facet Mindfulness Questionnaire and its short forms. Mindfulness, 10, 1615-1628. doi:10.1007/s12671-01901105-X

Petrocchi, N., \& Ottaviani, C. (2016). Mindfulness facets distinctively predict depressive symptoms after two years: The mediating role of rumination. Personality and Individual Differences, 93, 92-96. doi:10.1016/j.paid.2015.08.017

Podskaoff, P. M., MacKenzie, S. B., Lee, J-Y, \& Podskaoff, N. P. (2003). Common method biases in behavioural research: A critical review of the literature and recommended remedies. Journal of Applied Psychology, 88(5), 879-903. doi:10.1037/00219010.88 .5 .879

Rauch, W. A., Schweizer, K., \& Moosbrugger, H. (2007). Method effects due to social desirability as a parsimonious explanation of the deviation from unidimensionality in LOT-R scores. Personality and Individual Differences, 42(8), 1597-1607. doi:10.1016/j.paid.2006.10.035 
Reise, S. P. (2012). The rediscovery of bifactor measurement models. Multivariate Behavioral Research, 47(5), 667-696. doi:10.1080/00273171.2012.715555

Reise, S. P., Kim, D. S, Mansolf, M., \& Widaman, K. F. (2016). Is the bifactor model a better model or is it just better at modelling implausible responses? Application of iteratively reweighted least squares to Rosenberg Self-Esteem Scale. Multivariate Behavioral Research, 51(6), 818-838. doi:10.1080/00273171.2016.1243461

Rindskopf, D., \& Tedd, R. (1988). Some theory and application of confirmatory second-order factor analysis. Multivariate Behavioral Research, 23(1), 51-67. doi:10.1207/s15327906mbr2301_3

Roemer, L., Williston, S. K., \& Rollins, L. G. (2015). Mindfulness and emotion regulation. Current Opinion in Psychology, 3, 52-57. doi:10.1016/j.copsyc.2015.02.006

Rosseel, Y. (2012). lavaan: An R Package for Structural Equation Modeling. Journal of Statistical Software, 48(2), 1-36.

Soler, J., Cebolla, A., Feliu-Soler, A., Demarzo, M. M. P., Pascual, J. C., Baños, R., \& GarcíaCampayo, J. (2014). Relationship between meditative practice and self-reported mindfulness: The MINDSENS composite index. PLoS One, 9(1), e86622. doi:10.1371/journal.pone.0086622

Soto, C. J., \& John, O. P. (2017). Short and extra-short forms of the Big Five Inventory-2: The BFI-2-S and BFI-2-XS. Journal of Research in Personality, 68, 69-81. doi:10.1016/j.jrp.2017.02.004

Tran, U.S., Glück, T. M., \& Nader, I. W. (2013). Investigating the Five Facet Mindfulness Questionnaire (FFMQ): Construction of a short form and evidence of a two-factor higher 
order structure of mindfulness. Journal of Clinical Psychology, 69(9), 961-965. doi:10.1002/jclp.21996

Truong, Q. C., Krägeloh, C. U., Siegert, R. J., Landon, J., \& Medvedev, O. N. (2020). Applying generalizability theory to differentiate between trait and state in the Five Facet Mindfulness Questionnaire (FFMQ). Mindfulness, 11, 953-963. doi:10.1007/s12671-02001324-7

Tsai, J.L. (2007). Ideal affect: Cultural causes and behavioural consequences. Perspectives on Psychological Science, 2, 242-259.

Van Dam, N. T., Bilgrami, Z. R., \& Eisenlohr-Moul, T. (2018). Development and validation of the Balanced Inventory of Mindfulness-Related Skills (BIMS). MindRXiv.

\section{https://mindrxiv.org/8qdzg/}

Van Dam, N. T., Earleywine, M., \& Danoff-Burg, S. (2009). Differential item function across meditators and non-meditators on the Five Facet Mindfulness Questionnaire. Personality and Individual Differences, 47, 516-521. doi:10.1016/j.paid.2009.05.005

Van Dam, N. T., Hobkirk, A. L., Danoff-Burg, S., \& Earleywine, M. (2012). Mind your words: positive and negative items create method effects on the Five Facet Mindfulness Questionnaire. Assessment, 19(2), 198-204. doi:10.1177/1073191112438743

Van Dam, N. T., van Vugt, M. K., Vago, D. R., Schmalzl, L., Saron, C. D., Olendzki, A., ... Meyer, D. E. (2018). Mind the hype: A critical evaluation and prescriptive agenda for research on mindfulness and meditation. Perspectives on Psychological Science, 13(1), 36-61. doi:10.1177/1745691617709589

Veehof, M. M., ten Klooster, P. M., Taal, E., Westerhof, G. J., \& Bohlmeijer, E. T. (2011). Psychometric properties of the Dutch Five Facet Mindfulness Questionnaire (FFMQ-D) 
in patients with fibromyalgia. Journal of Clinical Rheumatology, 30(8), 1045-1054. doi:10.1007/s10067-011-1690-9

Vøllestad, J., Siversten, B., \& Nielsen, G. H. (2011). Mindfulness-based stress reduction for patients with anxiety disorders: Evaluation in a randomized controlled trial. Behaviour Research and Therapy, 49(4), 281-288. doi:10.1016/j.brat.2011.01.007

Williams, M. J., Dalgleish, T., Karl, A., \& Kyuken, W. (2014). Examining the factor structures of the Five Facet Mindfulness and the Self-Compassion Scale. Psychological Assessment, 26(2), 407-418. doi:10.1037/a0035566

Xia, Y., \& Yang, Y. (2019). RMSEA, CFI, and RLI in structural equation modeling with ordered categorical data: The story they tell depends on estimation methods. Behavior Research Methods, 51, 409-428. doi:10.3758/s13428-018-1055-2 


\section{Appendix}

Balanced Inventory of Mindfulness-related Skills (BIMS)

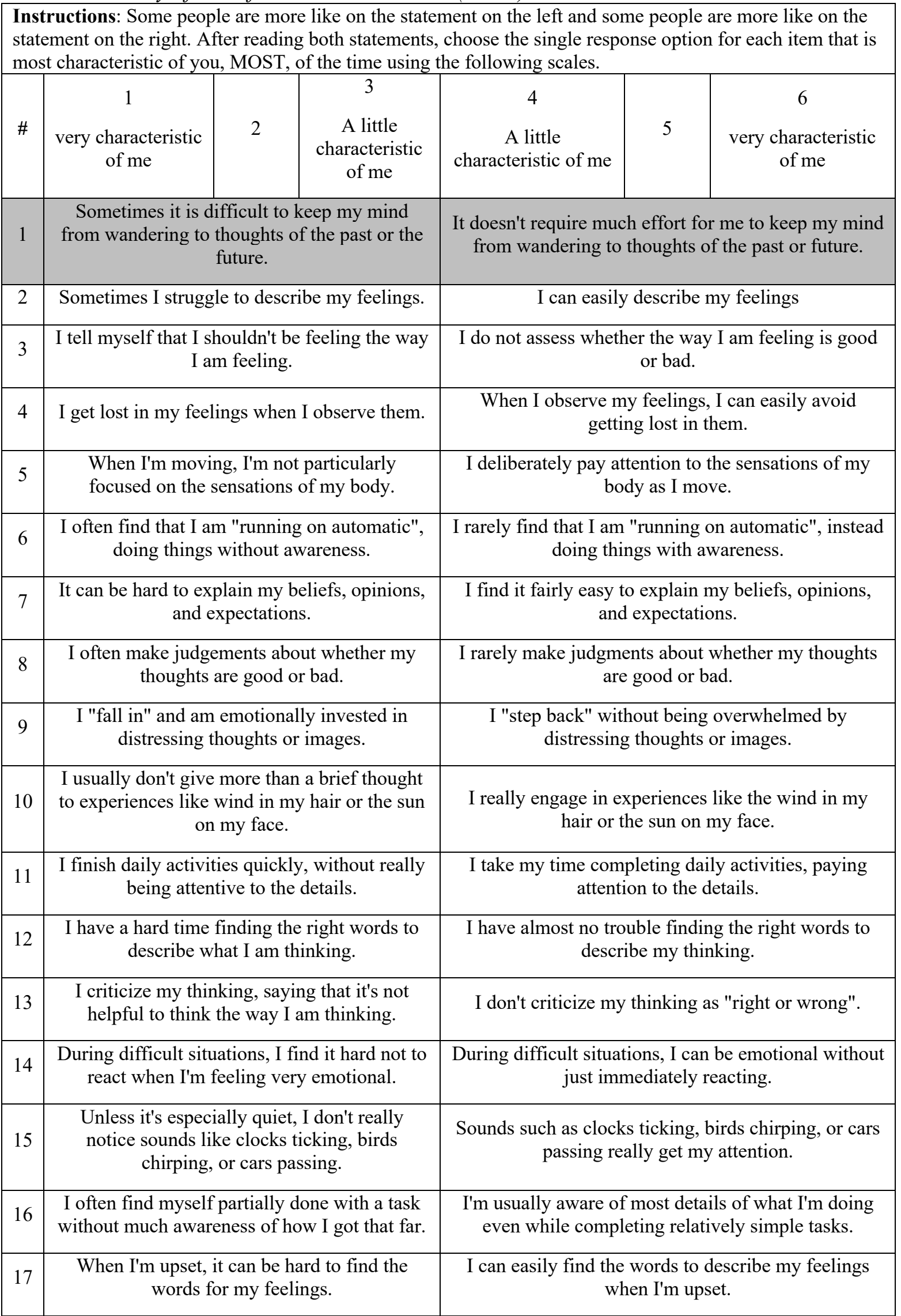




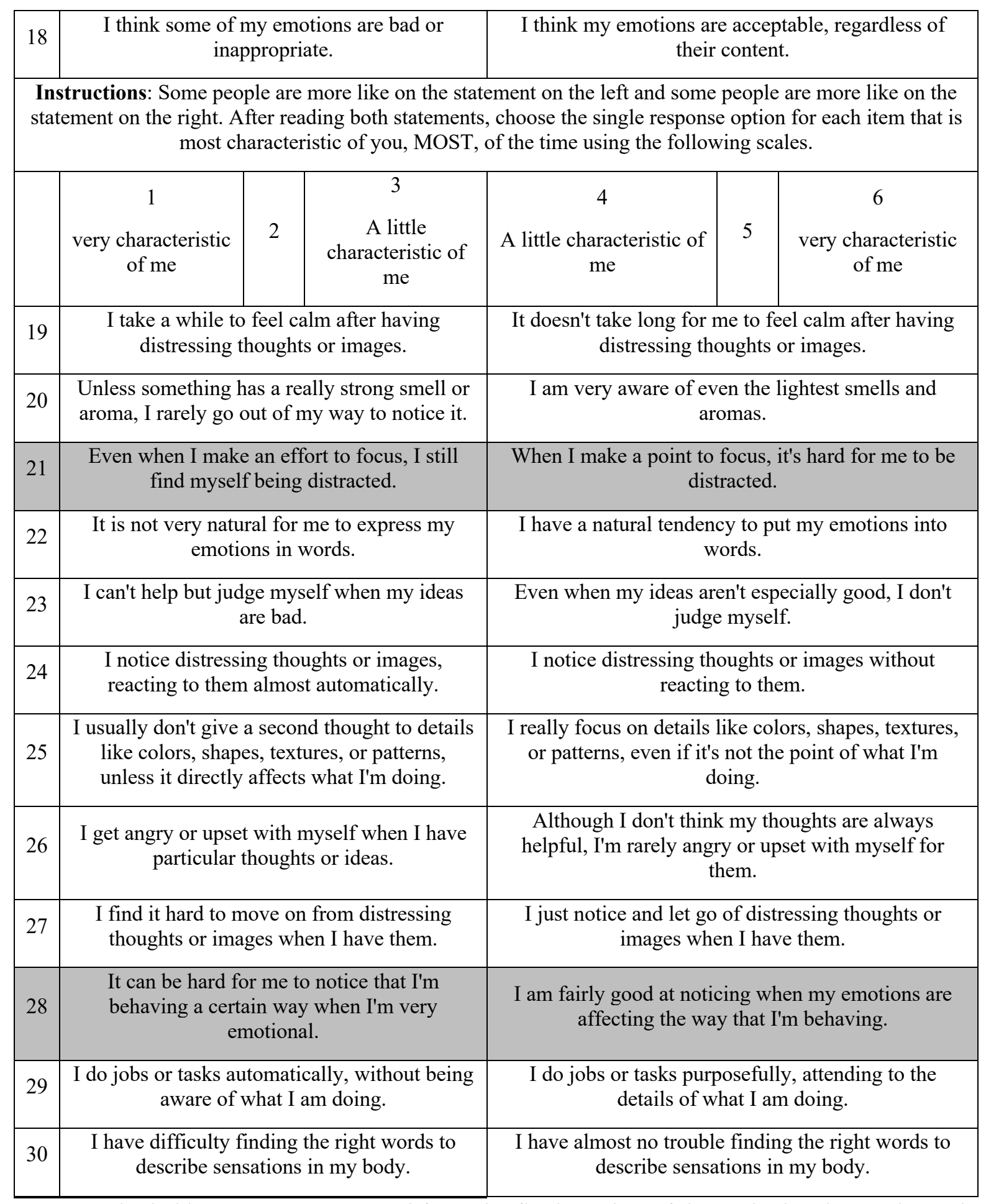

$N . B$. Items shaded in gray were removed from the final version of the scale, leaving 27 items in total.

Scoring is as follows:

ActAware $=\operatorname{sum}(6,11,16,29)$

Describe $=\operatorname{sum}(2,7,12,17,22,30)$

NonJudge $=\operatorname{sum}(3,8,13,18,23,26)$

NonReact $=\operatorname{sum}(4,9,14,19,24,27)$

Observe $=\operatorname{sum}(5,10,15,20,25)$

NOTE: There is no valid total score for the BIMS, and it should not be calculated or used. 